\title{
EXTRAOCULAR LIGHT EXPOSURE DOES NOT SUPPRESS PLASMA MELATONIN IN HUMANS
}

\author{
STEVEN W LOCKLEY, DEBRA J SKENE ${ }^{1}$, KAVITA THAPAN, JUDIE ENGLISH, DAVID \\ RIBEIRO, IRIS HAIMOV, SHELAGH HAMPTON, BENITA MIDDLETON, MALCOLM \\ von SCHANTZ, AND JOSEPHINE ARENDT
}

School of Biological Sciences, University of Surrey, Guildford, Surrey GU2 5XH, UK

\begin{abstract}
Light affects the circadian axis in at least two ways. It can cause the acute suppression of pineal melatonin synthesis, and/or a phase-shift of the circadian oscillator. As recent evidence has suggested that extraocular light exposure may cause phase-shifts of the circadian clock, we have investigated whether suppression of melatonin can be induced by the same type of light exposure. In the first study subjects' eyes were exposed to white light (2250 lux for $30 \mathrm{mins}$ ) via a fibre optic cable. As expected, suppression of nighttime plasma melatonin levels $(61 \pm 6 \%)$ was observed. In the second study, light of the same quality but higher intensity (14,000 or 67,500 lux for 180 mins) was delivered in the same manner to the popliteal region behind the subjects' knees, whilst shielding their eyes. No suppression of plasma melatonin levels $(4 \pm 7 \%)$ was detected in any of the subjects. Thus, extraocular photoreception, if it exists in mammals, does not affect the suprachiasmatic nucleipineal pathway.
\end{abstract}

Light is the most important stimulus for the entrainment of circadian rhythms in multicellular, and in some unicellular, organisms. In mammals, the circadian response to light is mediated through the lateral eyes.

Light affects the circadian axis in at least two ultimately interconnected ways. Firstly, it causes the acute suppression of pineal melatonin synthesis $(1,2)$. Secondly, it causes an enduring phase-shift of the circadian oscillator $(3,4)$. In humans (5) and in other mammals $(2,6,7)$, both of these effects are abolished by ocular enucleation. However, light-induced melatonin suppression has been reported in human subjects who are clinically blind (5). Indeed, experiments with animal models have indicated that neither rods nor cones appear to be required for photoentrainment at their respective absorption peaks $(7,8)$. Similarly, in humans, light-induced melatonin sup-

${ }^{1}$ To whom all correspondence should be addressed pression is not affected by red-green colour blindness (9).

The concept of ocular photoentrainment in mammals has recently been challenged by Campbell and Murphy (10). They exposed human subjects to 13,000-lux light pulses applied to the popliteal region of the knee, after which phase-shifts were observed both in core body temperature and in salivary melatonin levels. This remarkable observation, which presents a fundamental challenge to the current paradigm of photoreception in mammals, has led us to investigate if extraocular light exposure could also suppress nocturnal plasma melatonin levels.

\section{MATERIALS AND METHODS}

Two studies were conducted to assess the ability of broad spectrum white light to suppress plasma melatonin; Study 1 tested ocular exposure and Study 2 investigated extraocular (popliteal) exposure.

Ethical permission for the studies was granted by the University of Surrey Advisory Committee on Ethics and all subjects gave informed consent. Prior to the study, subjects collected sequential four-hourly urine samples ( $8 \mathrm{~h}$ overnight) for radioimmunoassay 
(RIA) analysis (11) of the major urinary metabolite of melatonin, 6-sulphatoxymelatonin (aMT6s). Subjects also wore Actillumes (Ambulatory Monitoring Inc., New York, NY) recording activity and light exposure continuously for 2-5 days (1-minute data epochs) before and during the study.

Both studies lasted for two nights and were carried out at the Clinical Investigation Unit (CIU) at the University of Surrey. All the subjects were male, healthy and drug free. They were asked to avoid bright sunlight and strenuous activity and refrain from caffeine- and alcohol-containing drinks between the two nights. Nine subjects took part. Two subjects completed both studies; one of them completed both light intensities in Study 2.

\section{Study 1}

Four males, all aged 18 years, were studied. Subjects entered the CIU before $19.00 \mathrm{~h}$ and had indwelling cannulae inserted. Blood samples were taken for measurement of plasma melatonin prior to, during and following light exposure (see Figs 1 and 2 for sampling times). Core body temperature (CBT) was measured continuously with a rectal temperature probe (Grant Instruments Ltd., Cambridge, UK) every 5 minutes. Environmental light conditions were as follows: 19.00-23.00 h, constant dim light $(<10$ lux); 23.00-07.00h, constant darkness and wearing eye-masks; 07.00-10.00 h, constant $\operatorname{dim}$ light $(<10$ lux). White light was generated by an are lamp, UV and IR filtered (Enlightened Technologies Associates Inc., Fairfax, USA) and delivered via a fibre optic bundle to two light rings (diameter $2.5 \mathrm{~cm}$ ). The rings were fitted into optician's trial frames so that the light rings were $2.5 \mathrm{~cm}$ from the front of the eye. Light (solar colour temperature $5830 \mathrm{~K}, 2250 \mathrm{lux}, 14 \mathrm{~W} / \mathrm{m}^{2}$, measured $2.5 \mathrm{~cm}$ from the light rings) was administered for 30 minutes $00.30-01.00 \mathrm{~h}(\mathrm{n}=2)$ or 01.15-01.45 $h(n=2)$ on Night 2 (N2). The experimental conditions were identical on both nights, with subjects in a semi-recumbent position from 19.00$23.00 \mathrm{~h}$ and $07.00-10.00 \mathrm{~h}$ and, lying supine with eye masks on 23.00-07.00 $\mathrm{h}$ except during the light exposure. On both nights, volunteers were moved to a chair 15 minutes before the start of the light exposure and fitted with the spectacles. During the exposure period, subjects were instructed to look straight ahead, keep their eyes fully open and move their eyes and head as little as possible. On N1 the light source was switched on and covered. The subjects wore the spectacles and light rings but the fibre optic cables were not attached to the light source.

\section{Study 2}

Seven males aged 18-31 years were studied, one of whom completed two light intensities. Plasma melatonin and CBT measurements were as in Study 1. Environmental light conditions were as follows: 21.00-23.00 h constant dim light ( $<10 \mathrm{lux})$; 23.00$07.00 \mathrm{~h}$ constant darkness and wearing eye-masks; 07.00-08.00 h constant dim light ( $<10$ lux). Using the same light source as Study 1 and in a single blind design, light of two intensities $\left(14,000 \mathrm{lux}, 90 \mathrm{~W} / \mathrm{m}^{2}\right.$ and $67,500 \mathrm{lux}, 430 \mathrm{~W} / \mathrm{m}^{2}$, measured $2 \mathrm{~mm}$ from the light rings) was administered for 180 minutes 24.00$03.00 \mathrm{~h}$ on $\mathrm{N} 2$ ( $\mathrm{n}=4$ per intensity). A light ring was strapped to the back of each knee (popliteal region). Subjects remained supine for the entire 23.00-07.00 h period and lay under a duvet. They were kept awake before and during the exposure. On N1, the light source was switched on and covered. The subjects had the light rings strapped behind each knee but the fibre optic cables were not attached to the light source.

\section{Melatonin RLA}

Blood samples were centrifuged and plasma stored at $-20^{\circ} \mathrm{C}$ until analysis by RIA (12). The limit of detection of the assay was $2.8 \mathrm{pg} / \mathrm{ml}$. The samples from each subject were measured in the same assay.

All errors are expressed as standard deviation. Suppression of melatonin was calculated from the difference in melatonin levels between $\mathrm{N} 1$ and N2 for the samples collected at and 15 minutes after lights off. Statistical significance was calculated using paired Student's t-test.

\section{RESULTS}

Prior to the studies, assessment of urinary aMT6s confirmed that each subject had a normal aMT6s rhythm with a night-time peak or acrophase (mean 24 h aMT6s production $=14.8 \pm 3.8 \mu \mathrm{g} / 24 \mathrm{~h}$; mean acrophase $=4.8 \pm 2.2 \mathrm{~h}$ ).

\section{Study 1}

Three of the four subjects showed a significant suppression in plasma melatonin levels during and following a 30-minute ocular exposure to $2,250 \mathrm{lux}\left(14 \mathrm{~W} / \mathrm{m}^{2}\right)$ white light (Fig. 1). The mean suppression was $61 \pm$ $6 \%(\mathrm{n}=3)$. Melatonin was maximally suppressed 30 and 45 minutes after lights on (Fig. 1). Plasma melatonin concentrations returned to N1 levels 


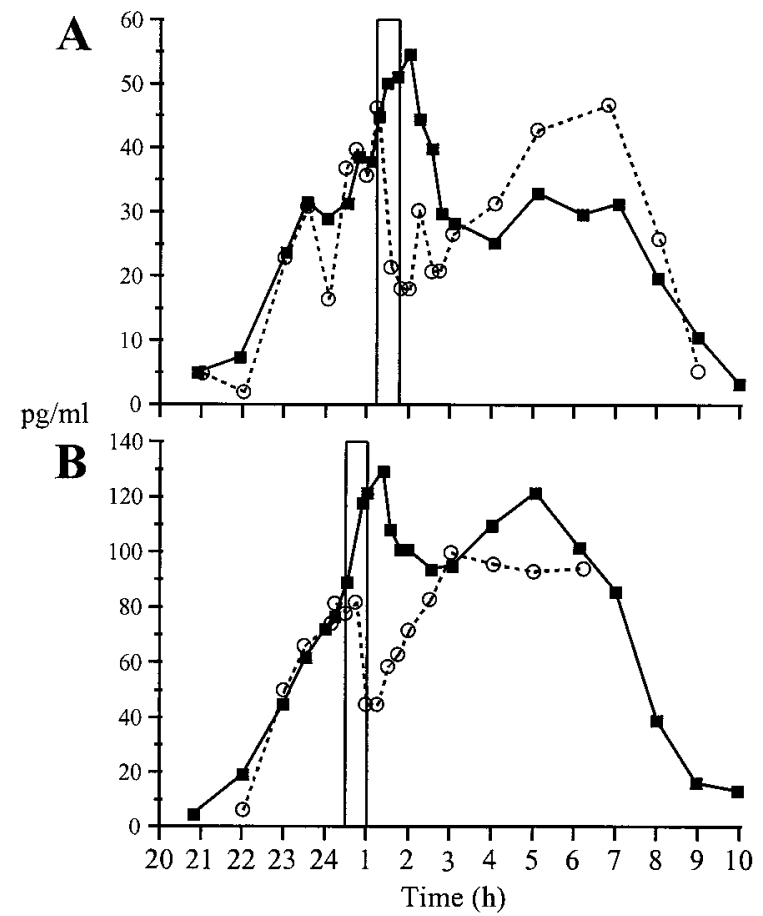

Figure 1. Ocular light exposure in two subjects. Plasma melatonin was suppressed during and following 30 minutes of ocular light exposure (open bars, 2250 lux) on N2 (O, dotted line) when compared to the control night (N1; $\mathbf{0}$, solid line).

within $106 \pm 14$ minutes after lights off. The one subject who did not show suppression had an unusual melatonin rhythm on both nights, with a low amplitude peak at $23.00 \mathrm{~h}$ $(30 \mathrm{pg} / \mathrm{ml})$, and gradually falling over the rest of the night. This subject also completed study $2(67,500$ lux exposure), and a similar melatonin rhythm was observed on both nights. He was excluded from any analysis.

\section{Study 2}

There was no evidence of any suppression of plasma melatonin levels during and following 180 minutes of popliteal light exposure of $14,000 \mathrm{lux}\left(90 \mathrm{~W} / \mathrm{m}^{2}\right)$ and 67,500 $\operatorname{lux}\left(430 \mathrm{~W} / \mathrm{m}^{2}\right)$ (Fig. $2 \mathrm{~A}$ and $2 \mathrm{~B}$, respectively). The mean suppression was $5 \pm 8 \%$ and $3 \pm$ $7 \%$ for $14,000 \operatorname{lux}(n=4)$ and $67,500 \operatorname{lux}(n=3)$, respectively, amounting to $4 \pm 7 \%(\mathrm{n}=7)$ overall. The one subject that completed both studies and both light intensities for Study 2 showed suppression (65\%) following ocular exposure (Fig. 1A) but no suppression at either intensity following popliteal exposure.

The Actillume recordings confirmed that the subjects kept a regular sleep-wake and activity schedule and were not inappropriately exposed to light. The similar melatonin onset and offset times observed on both study nights also support the idea that no phase shift of the circadian clock occurred during the study period.

In neither study did light exposure acutely affect CBT. There was no significant difference between N1 and N2 at lights off.

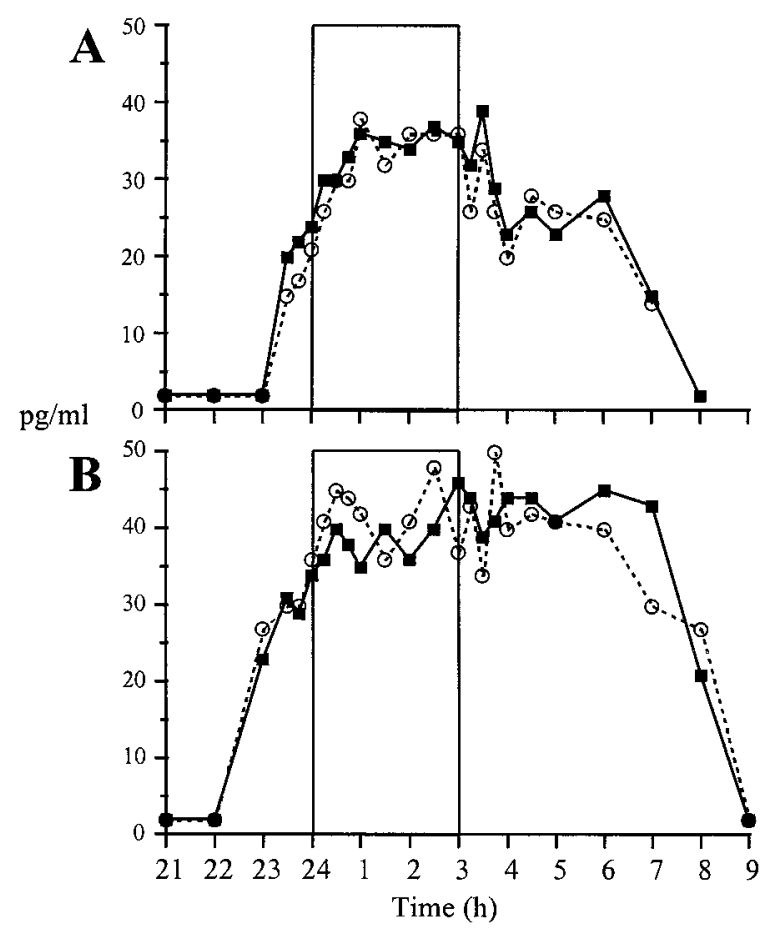

Figure 2. Extraocular (popliteal) light exposure in two subjects. Plasma melatonin was not suppressed during and following 180 minutes of extraocular light exposure (open bars) at either 14,000 lux (Fig. 2A) or 67,500 lux (Fig. 2B) on N2 (O, dotted line) when compared to the control night (N1; $\mathbf{\square}$, solid line). 


\section{DISCUSSION}

The inability of popliteal light exposure to suppress the nocturnal plasma melatonin rhythm was in sharp contrast to the effect of ocular light exposure administered in the same way. Inhibition of melatonin production by ocular illumination (as well as the neural pathways involved) has been well documented and the effect is known to depend on the intensity, duration and wavelength of light. Our results support the established view that intact, uncovered eyes are a prerequisite for light-induced suppression of melatonin in humans.

The subjects avoided inappropriate exposure to bright light before and during the study in order to prevent a phase shift of the circadian clock during the study period. The lack of phase shift was confirmed by the consistent timing of the melatonin rhythm. Changes in the melatonin concentrations observed following ocular exposure are thus a result of acute suppression by light.

The intensity of light used in this report $(67,500$ lux $)$ exceeds that utilised in any previous study. More intense ocular illumination is required to produce a phaseshift than to suppress melatonin synthesis $(13,14)$. Thus, apart from methodological differences, the only way in which the data presented here could be reconciled with those of Campbell and Murphy (10) is if these effects of light are mediated through different photoreceptors and/or pathways. We conclude that popliteal light exposure and its proposed mechanism of "humoral phototransduction" (15) do not affect the suprachiasmatic nuclei-pineal pathway.

\section{References}

1. Lewy AJ, Wehr TA, Goodwin FK, Newsome DA, Markey SP 1980 Light suppresses melatonin secretion in humans. Science 210:1267-1269

2. Klein DC, Weller JL 1972 Rapid light-induced decrease in pineal serotonin $\mathrm{N}$-acetyltransferase activity. Science $177: 532-533$
3. Arendt J, Broadway J 1986 Phase response of human melatonin rhythms to bright light in Antarctica. J Physiol 377:68P

4. Czeisler CA, Allan JS, Strogatz SH, et al. 1986 Bright light resets the human circadian pacemaker independent of the timing of the sleep-wake cycle. Science 233:667-671

5. Czeisler CA, Shanahan TL, Klerman EB, et al. 1995 Suppression of melatonin secretion in some blind patients by exposure to bright light. $N$ Engl $J$ Med 332:6-11

6. Nelson RJ, Zucker I 1981 Absence of extraocular photoreception in diurnal and nocturnal rodents exposed to direct sunlight. J Comp Biochem Physiol 69A: $145-148$

7. Foster RG, Provencio I, Hudson D, et al. 1991 Circadian photoreception in the retinally degenerate mouse $(r d / r d)$. J Comp Physiol A 169:39-50

8. von Schantz M, Argamaso-Hernan SM, Szél Á, Foster RG 1997 Photopigments and photoentrainment in the Syrian golden hamster. Brain Res 770:131-138

9. Ruberg FL, Skene DJ, Hanifin JP, et al. 1996 Melatonin regulation in humans with color vision deficiencies. J Clin Endocrinol Metab 81:2980-2985 10. Campbell SS, Murphy PJ 1998 Extraocular circadian phototransduction in humans. Science 279:396-39

11. Aldhous ME, Arendt J 1988 Radioimmunoassay for 6-sulphatoxymelatonin in urine using an iodinated tracer. Ann Clin Biochem 25:298-303

12. Fraser S, Cowen P, Franklin M, Franey C, Arendt J 1983 Direct radioimmunoassay for melatonin in plasma. Clin Chem 29:396-397

13. Nelson DE, Takahashi JS 1991 Sensitivity and integration in a visual pathway for circadian entrainment in the hamster (Mesocricetus auratus). J Physiol Lond 439:115-45

14. Hashimoto S, Nakamura K, Honma $\mathbf{S}$, Tokura H, Honma K-I 1996 Melatonin rhythm is not shifted by lights that suppress melatonin in humans under entrainment. Am J Physiol 39:R1073-R1077

15. Oren DA, Terman M 1998 Tweaking the human circadian clock with light. Science 279:333334

\section{Acknowledgements}

This work was supported by The Wellcome Trust (Grant 048197/Z/96/Z) and Stockgrand Ltd., UK. The authors wish to thank Ms. Selvamalar Ratnasingam for invaluable help with sample collection. 